\title{
Surgical management of colorectal liver metastases
}

\author{
Keith Michael Cavaness*1 \\ ${ }^{1}$ Department of Surgical Oncology/Hepatobiliary Surgery, Baylor University Medical Center, Dallas, TX 75246, USA
}

Colorectal cancer is the third most commonly diagnosed cancer among men and women worldwide. Of the 1.2 million projected new cases in 2017, approximately half will develop metastases to the liver [1,2]. The incidence of colorectal cancer among those less than 50 years old continues to rise, with a reported increase of $22 \%$ from $2000-2013$ [3]. It has been estimated that over half of the patients who die of colorectal cancer have liver metastases at autopsy, while $35 \%$ have isolated hepatic metastases. Metastatic liver disease is the cause of death in most patients [4]. Currently, hepatic resection is considered the treatment of choice for patients with colorectal liver metastasis (CLM), demonstrating a 5-year survival of $35-60 \%[5,6]$.

Management strategies can vary widely when discussing the treatment of liver metastases from colorectal cancer. Conflicting strategies include the use of neoadjuvant chemotherapy, the timing of resection for synchronous metastases, how to approach disappearing liver lesions, the best methods to treat bilobar disease, the treatment of recurrent metastases or the indications for surgery in extrahepatic disease [7]. Managing patients with metastatic disease is best achieved through a multidisciplinary approach. At our center, patients are presented at a multidisciplinary tumor conference where detailed discussion helps focus on the goals of treatment. Through interactions with surgeons, oncologists, pathologists, radiologists and key ancillary staff, an optimal treatment plan can be established.

\section{Patient selection}

The morbidity associated with liver resection is directly linked to patient selection. Although multiple factors are considered in surgical decision making, studies have shown that postoperative complications are significantly affected by the American Society of Anesthesiologists (ASA) score, the presence of hepatic steatosis, the extent of resection, and an associated extrahepatic procedure [8]. Therefore, the patient's clinical performance status and comorbidities should allow them to undergo a major resection with an expected mortality risk of less than $5 \%$.

\section{Imaging}

Recent studies have compared the efficacy of gadoxetic acidenhanced MRI (EOVIST) to conventional MRI and Contrast enhanced CT (CECT), in detecting colorectal liver metastases [9]. They concluded that EOVIST MRI was superior as a first line imaging modality in detecting CLM. While this modality may showed improved detection, it is often not available at all institutions. Where EOVIST MRI is not available, a CECT that includes arterial, portal venous and delayed venous phases can effectively help to define the burden of disease, relevant anatomy and aid in pre-operative planning.

\section{Techniques for resection}

Historically, subgroups of patients with multiple tumors, large tumor size, lymph node involvement, or extrahepatic disease were considered unresectable. However, the use of newer chemotherapy protocols has proven effective in reducing the tumor burden while observing the tumor response known as the "biology of the tumor". This technique better prepares the patient for curative resection. The major goal of resection is to remove all metastatic disease with negative margins.

The extent of hepatic involvement significantly affects patient selection and the ideal treatment strategy. The future liver remnant (FLR) volume must be healthy enough to carry out hepatic function in the postoperative period. If chemotherapy induced liver injury or cirrhosis is present, the allowable extent of resection is reduced dramatically, and ablation may assume a larger therapeutic role. If the anticipated FLR is considered marginal, strategies utilizing hepatic regeneration can transform some patients from unresectable to resectable. Portal vein embolization (PVE), staged resection and in-situ staged resection (ALPPS) are three treatment modalities that can help to accomplish this goal $[10,11]$.

Widespread bilobar metastases require careful planning for the best approach. These cases are frequently considered unresectable however with a combination of resection and non-resectional techniques, all liver disease can often be addressed. Involvement of the afferent or efferent vasculature may require advanced vascular resections while involvement of both the afferent and efferent vasculature may be a contraindication to resection.

\section{Conclusion}

The management of CLM has evolved over the past decade. With accurate staging and targeted therapies, a 5 -year survival of $>40 \%$ can be expected [6]. Patient selection is crucial and requires candidates to tolerate a major laparotomy with an expected mortality $<5 \%$. The goals of surgical management should include a margin negative resection with the preservation of adequate FLR volume. Minimal future liver remnant (FLR) volume has been set at $>20 \%$ for a normal liver, $>30 \%$ in a damaged liver after extensive treatment, and $>40 \%$ for a cirrhotic liver [12]. Strategies for improving resection candidacy include PVE, two-staged hepatectomy, neoadjuvant chemotherapy to assess the tumor biology and local ablative techniques that control the disease burden while preparing for resection.

Correspondence to: Keith Michael Cavaness, DO, FACS, Department of Surgical Oncology/Hepatobiliary Surgery, Baylor University Medical Center, Dallas, TX 75246, USA, E-mail: Keith.Cavaness@BSWHealth.org

Key words: liver resection, portal vein embolization, alpps, colorectal liver metastasis, colorectal cancer, EOVIST MRI.

Received: April 09, 2017; Accepted: April 19, 2017; Published: April 21, 2017 


\section{References}

1. Moris D, Dimitroulis D, Vernadakis S (2017) Parenchymal-sparing hepatectomy as the new doctrine in the treatment of liver-metastatic colorectal disease: beyond oncological outcomes. Anticancer Res 37: 9-14.

2. Yoo PS, Lopez-Soler RI, Longo WE, Cha CH (2006) Liver resection for metastatic colorectal cancer in the age of neoadjuvant chemotherapy and bevacizumab. Clin Colorectal Cancer 6: 202-207. [Crossref]

3. Siegel RL, Miller KD, Fedewa SA, Ahnen DJ, Meester RG, et al. (2017) Colorectal cancer statistics, 2017. CA Cancer J Clin. [Crossref]

4. Foster JH (1984) Treatment of metastatic disease of the liver: a skeptic's view. Semin Liver Dis 4: 170-179. [Crossref]

5. de Haas RJ, Wicherts DA, Andreani P, Pascal G, Saliba F, et al. (2011) Impact of expanding criteria for resectability of colorectal metastases on short- and long-term outcomes after hepatic resection. Ann Surg 253: 1069-1079. [Crossref]

6. Choti MA, Sitzmann J V, Tiburi MF, Sumetchotimetha W, Rangsin R, et al. (2002) Trends in long-term survival following liver resection for hepatic colorectal metastases. Ann Surg 235: 759-766. [Crossref]
7. Minagawa M, Yamamoto J, Kosuge T, Matsuyama Y, Miyagawa S-I, et al. (2007) Simplified staging system for predicting the prognosis of patients with resectable liver metastasis: development and validation. Arch Surg 142: 269-276. [Crossref]

8. Belghiti J, Hiramatsu K, Benoist S, Massault P, Sauvanet A, et al. (2000) Seven hundred forty-seven hepatectomies in the 1990s: an update to evaluate the actual risk of liver resection. J Am Coll Surg 191: 38-46. [Crossref]

9. Zech CJ, Korpraphong P, Huppertz A, et al. (2014) Randomized multicentre trial of gadoxetic acid-enhanced MRI versus conventional MRI or CT in the staging of colorectal cancer liver metastases. Br J Surg 101: 613-621. [Crossref]

10. Abulkhir A, Limongelli P, Healey AJ, Damrah O, Tait P, et al. (2008) Preoperative portal vein embolization for major liver resection: a meta-analysis. Ann Surg 247: 4957. [Crossref]

11. Torzilli G, Adam R, Viganò L, Imai K, Goransky J, et al. (2016) Surgery of Colorectal Liver Metastases: Pushing the Limits. Liver Cancer 6: 80-89. [Crossref]

12. Shindoh J, Tzeng CW, Aloia TA, Curley SA, Zimmitti G, et al. (2013) Optimal future liver remnant in patients treated with extensive preoperative chemotherapy for colorectal liver metastases. Ann Surg Oncol 20: 2493-2500. [Crossref]

Copyright: $@ 2017$ Cavaness KM. This is an open-access article distributed under the terms of the Creative Commons Attribution License, which permits unrestricted use, distribution, and reproduction in any medium, provided the original author and source are credited. 